\title{
Deep Sign: Enabling Robust Statistical Continuous Sign Language Recognition via Hybrid CNN-HMMs
}

\author{
Oscar Koller ${ }^{1}$ D $\cdot$ Sepehr Zargaran ${ }^{1} \cdot$ Hermann Ney $^{1} \cdot$ Richard Bowden $^{2}$
}

Received: 1 February 2017 / Accepted: 3 September 2018 / Published online: 5 October 2018

(c) The Author(s) 2018

\begin{abstract}
This manuscript introduces the end-to-end embedding of a CNN into a HMM, while interpreting the outputs of the CNN in a Bayesian framework. The hybrid CNN-HMM combines the strong discriminative abilities of CNNs with the sequence modelling capabilities of HMMs. Most current approaches in the field of gesture and sign language recognition disregard the necessity of dealing with sequence data both for training and evaluation. With our presented end-to-end embedding we are able to improve over the state-of-the-art on three challenging benchmark continuous sign language recognition tasks by between 15 and $38 \%$ relative reduction in word error rate and up to $20 \%$ absolute. We analyse the effect of the CNN structure, network pretraining and number of hidden states. We compare the hybrid modelling to a tandem approach and evaluate the gain of model combination.
\end{abstract}

Keywords Sign language recognition · Hybrid approach $\cdot$ CNN-HMM $\cdot$ Statistical approach $\cdot$ Sequence modelling

\section{Introduction}

Face-to-face communication is often the preferred choice, when either important matters need to be discussed or informal links between individuals are established. Gesture is a key part in such human-to-human communication. It helps us to better understand the other party. However, the role of visual cues in spoken language is not well defined. As such, the task of gesture recognition is also not accurately defined. This renders comparison of algorithms and approaches difficult. Sign language on the other hand provides a clear framework with a defined inventory and grammatical rules that govern joint expression by hand (movement, shape, orientation, place of articulation) and by face (eye gaze, eye brows, mouth, head orientation). This makes sign languages, the natural languages of the deaf, a perfect test bed for computer vision and human

Communicated by Edwin Hancock, Richard Wilson, Will Smith, Adrian Bors and Nick Pears.

Oscar Koller

koller@cs.rwth-aachen.de

1 Human Language Technology and Pattern Recognition, RWTH Aachen University, Aachen, Germany

2 Centre for Vision Speech and Signal Processing, University of Surrey, Guildford, UK language modelling algorithms targeting human computer interaction and gesture recognition. The rules governing the interaction of hands and body, referred to as the manual and non-manual parts - are well defined by sign language theory. Videos represent a time series of dynamic images and the recognition of sign language therefore needs to be able to cope with variable input sequences and execution speed. Different schemes are followed to achieve this ranging from sliding window approaches (Ong et al. 2014) to temporal normalisations (Molchanov et al. 2015) or dynamic time warping (Krishnan and Sarkar 2015). While the field of automatic speech recognition is dominated by HiddenMarkov-Models (HMMs), they remain rather unpopular in computer vision related tasks. For instance CVPR, by many regarded as the top conference of computer vision, had only three out of a total of over 700 submissions in the year 2017 that were using HMMs (Koller et al. 2017; Richard et al. 2017; Schober et al. 2017). This may be related to the comparatively poor image modelling capabilities of Gaussian Mixture Models (GMMs), which had been traditionally used to model the observation probabilities within such a framework. More recently, deep Convolutional Neural Networks (CNNs) have outperformed other approaches in all computer vision tasks. In this work, we focus on integrating CNNs in a HMM framework, extending an interesting line 
of work (Koller et al. 2015b, 2016a; Le et al. 2015; Wu and Shao 2014), which we will discuss more closely in Sect. 2.

This manuscript presents the extended version of our previous work (Koller et al. 2016b), where we first presented a powerful embedding of a deep CNN in a HMM framework in the context of sign language and gesture recognition, while treating the outputs of the $\mathrm{CNN}$ as true Bayesian posteriors and training the system as a hybrid CNN-HMM in an end-toend fashion. With this method we are able to achieve a large relative improvement of over $15 \%$ compared to the state-ofthe-art on three challenging standard benchmark continuous sign language recognition data sets. In the scope of this extended manuscript, we make several additional contributions and have completely reran all experimental evaluation to allow us to provide more extensive results and deeper insights:

1. We significantly add to the theoretical explanation of the hybrid approach, with the aim of making its idea more accessible to newcomers to the field.

2. We analyse the effect of both CNN- and HMM-structure on the hybrid approach.

3. We investigate the effect of using out-of-domain data to train the network prior to finetuning using in-domain data.

4. We show that different training iterations provide complementary classifiers, which are able to further boost recognition when employed as ensembles of hybrid CNN-HMMs.

The rest of this manuscript is organised as follows: Sect. 2 discusses the related literature in depth. In Sect. 3 we introduce the theoretical basis of the presented hybrid approach. Differences w.r.t. the tandem approach are also described. The employed data sets are discussed in Sect. 4. Section 5 gives details on the implementation in order to ensure reproducibility, which is followed by the actual experimental evaluation in Sect. 6. Finally, we conclude the work in Sect. 7.

\section{Related Work}

After the recent success of CNNs (LeCun et al. 1998) in many computer vision fields, they have also shown large improvements in gesture and sign language recognition (Neverova et al. 2014; Huang et al. 2015; Koller et al. 2015b). However, in most previous CNN-based approaches the temporal domain of video data is not elegantly taken into consideration. Most approaches simply use a sliding window or circumvent the sequence properties by evaluating the output in terms of per-frame overlap with the ground truth, e.g. in Pigou et al. (2018). Moreover, CNNs are usually trained on the frame-level. A few artificial data sets such as the Mon- talbano gesture data set (Escalera et al. 2014) provide frame labels. However, this is usually not the case, especially for sign language footage or other real-life data sets. Available annotation usually consists of sequences of signs without explicit frame-level information. As such, the focus of the field needs to move towards approaches that deal with variable length inputs and outputs that do not require explicit frame labelling. The difficulty in accurately labelling single frames for evaluation further supports the need for such change. Graphical models such as HMMs lend themselves well to tasks with inputs of variable length. As will be shown in this work, we are able to combine the best of different worlds when integrating HMMs and CNNs. A few works have joined neural networks and HMMs before in the scope of gesture and sign language recognition. Wu and Shao (2014) use 3D CNNs to model the observation probabilities in a HMM. However, they interpret the CNN outputs as likelihoods $p(x \mid k)$ for an image $x$ and a given class $k$. Conversely, Richard and Lippmann (1991) showed that neural network outputs are better interpreted as posterior probabilities $p(k \mid x)$ in a Bayesian framework. In the field of speech recognition, Bayesian hybrid neural network HMMs were first proposed by Bourlard and Wellekens (1989) and became the approach of choice, particularly after the recent rise of deep learning. Le et al. (2015) followed this line of thought for gesture recognition, but only employed a shallow legacy neural network that was trained to distinguish twelve artificial actions. Koller et al. (2013, 2014) achieved important results using GMM-HMMs for weakly supervised learning in the domain of sign language. However, hybrid models strongly outperformed the results (Koller et al. 2016a), which constituted first and preliminary work in this direction. CNNs were employed in a hybrid Bayesian framework to perform weakly supervised training with the purpose of learning hand shape classifiers that generalise across data sets. The main differences with respect to this manuscript are that we learn the $\mathrm{CNN}$ top down using nothing more than the annotated sign-words (which are modelled by a fixed number of hidden states), whereas Koller et al. (2016a) models signs bottom up with additional knowledge of the decomposition of sign-words into different hand shapes which form the building blocks for signs. Moreover, in this work, we learn the $\mathrm{CNN}-\mathrm{HMM}$ in an end-to-end fashion from video input to gloss output, whereas in the previous work, the intermediate hand shape-CNN serves as feature extractor for an additional GMM-HMM sign model (similar to the tandem approach introduced in Sect. 3.3). In this so-called tandem modelling (refer to Sect. 3.3), the GMM-HMM needs to be completely retrained, which adds significant computational overhead. In the proposed hybrid approach, no GMM-retraining is necessary and in the experimental evaluation of this manuscript we will show that our approach clearly outperforms Koller et al. (2016a). Wu et al. (2016) published a paper that is also 
closely related to this work, but they do not interprete the CNN outputs in a Bayesian way, they use different inputs to the CNN (full body RGB and depth, as opposed to using a cropped hand patch) and different inputs to the HMM. Later, Granger and el Yacoubi (2017) provided a comparison between a hybrid neural network HMMs and a recurrent neural network (RNN) on a gesture task, finding that both perform comparably, while the state-based representation of the HMM allows better insights in the internals of the model for potential error analysis. Recently, Connectionist Temporal Classification (CTC) by Graves and Schmidhuber (2005) has received attention by the computer vision community in general (Assael et al. 2016; Cui et al. 2017; Rao et al. 2017). CTC is a training criterion for recurrent neural networks and very related to HMMs. CTC has been shown to be a special case of the hybrid full-sum HMM alignment with a specific HMM architecture. As such CTCs are related to this work. However, we do not use recurrent or long short term memory (LSTM) networks in this work. The interested reader may consult Bluche et al. (2015) for details on the comparison of CTC and HMMs.

Finally, this manuscript represents a more thorough version of Koller et al. (2016b), with much more extensive experiments. In addition, this manuscript analyses the effect of both CNN- and HMM-structure on the hybrid approach. It also investigates the effect of using out-of-domain data to pretrain the network prior to finetuning using in-domain data and the use of ensembles of CNN-HMMs in model combination to further boost performance. Koller et al. (2017) even drop the dependence on a hand tracking system and take the re-alignment of hybrid models for sign recognition further.

Another related approach has been introduced by Bengio and Frasconi (1996), where a RNN is used to extract temporally local information whereas a HMM integrates long-term constraints. The so-called input output HMM has been used by Marcel et al. (2000) in a basic gesture system that distinguishes between two gesture classes, deictic and symbolic.

\section{Continuous Sign Language Recognition}

The problem to be solved is a sequence learning task, which means we want to predict a sequence of output symbols $w_{1}^{N}$, in our case sign words (so-called "glosses", representing the semantics of the described word). Given an input video as a sequence of full images $X_{1}^{T}=X_{1}, \ldots, X_{T}$ and the resulting preprocessed (e.g. tracked and mean-normalised) images $x_{1}^{T}=x_{1}, \ldots, x_{T}$, automatic continuous sign language recognition tries to find an unknown sequence of sign-words $w_{1}^{N}$ for which $x_{1}^{T}$ best fit the learned models. We assume that images and sign-words occur in an ordered fashion. It has to be noted that this requirement clearly distinguishes the problem of sign language recognition from the problem of translating from sign language to spoken language where re-orderings are necessary and monotonicity cannot be assumed.

\subsection{Legacy GMM-HMM Approach}

To find the best fitting sequence, we follow the statistical paradigm (Bahl et al. 1983) using the maximum-a-posteriori simplification of Bayes' decision rule, which has been successfully applied to Automatic Speech Recognition (ASR), hand writing recognition and statistical machine translation since the early 1970s. Given a loss function $\mathcal{L}\left[w_{1}^{N}, \tilde{w}_{1}^{N}\right]$ between the true output sequence $w_{1}^{N}$ and the hypothesised output sequence $\tilde{w}_{1}^{N}$, Bayes' Decision Rule minimises the expected loss:

$$
x_{1}^{T} \rightarrow\left[w_{1}^{N}\right]_{\mathrm{opt}}=\underset{\tilde{w}_{1}^{N}}{\operatorname{argmin}}\left\{\sum_{w_{1}^{N}} \operatorname{Pr}\left(w_{1}^{N} \mid x_{1}^{T}\right) \cdot \mathcal{L}\left[w_{1}^{N}, \tilde{w}_{1}^{N}\right]\right\}
$$

Often Bayes Decision Rule is simplified to the maximuma-posteriori (MAP) rule, which is known to be equivalent for the case of the simple 0-1-loss.

$x_{1}^{T} \rightarrow\left[w_{1}^{N}\right]_{\mathrm{opt}}=\underset{w_{1}^{N}}{\operatorname{argmax}}\left\{\operatorname{Pr}\left(w_{1}^{N} \mid x_{1}^{T}\right)\right\}$

In sign language recognition the $0-1-$ loss corresponds to a minimisation of the expected sentence error rate, which counts an output sentence as wrong if a single recognised sign-word is wrong. However, for longer sentences, the sentence error rate does not correlate with the word error rate (WER) which is also known as edit distance and what we seek to minimise. As shown by Schlüter et al. (2012), the MAP rule is equivalent to the Bayes Rule for the WER as a loss function if

$\max _{w_{1}^{N}}\left\{\operatorname{Pr}\left(w_{1}^{N} \mid x_{1}^{N}\right)\right\}>0.5$

Therefore, we follow the MAP rule as the optimisation criterion and maximise the class posterior probability distribution $\operatorname{Pr}\left(w_{1}^{N} \mid x_{1}^{T}\right)$ over the whole utterance, as given in Eq. (2).

Decision theory allows us to split up the class posterior probability into the class prior $\operatorname{Pr}\left(w_{1}^{N}\right)$ and the class-conditional probability $\operatorname{Pr}\left(x_{1}^{T} \mid w_{1}^{N}\right)$, which can then be modelled by different information sources. The first term can be interpreted as word sequence knowledge which can be approximated by a n-gram language model estimating $p\left(w_{1}^{N}\right)$. The second term represents the actual visual knowledge, which historically used to be modelled by generative GMMs. 
$\left[w_{1}^{N}\right]_{\mathrm{opt}}=\underset{w_{1}^{N}}{\operatorname{argmax}}\left\{p\left(w_{1}^{N}\right) \cdot p\left(x_{1}^{T} \mid w_{1}^{N}\right)\right\}$

Expressing the class-conditional probability in terms of a HMM adds the hidden variable $s_{1}^{T}$ :

$$
\begin{aligned}
p\left(x_{1}^{T} \mid w_{1}^{N}\right) & =\sum_{s_{1}^{T}} p\left(x_{1}^{T}, s_{1}^{T} \mid w_{1}^{N}\right) \\
& =\sum_{s_{1}^{T}} \prod_{t=1}^{T} p\left(x_{t}, s_{t} \mid x_{1}^{t-1}, s_{1}^{t-1}, w_{1}^{N}\right) \\
& =\sum_{s_{1}^{T}} \prod_{t=1}^{T} p\left(x_{t} \mid x_{1}^{t-1}, s_{1}^{t}, w_{1}^{N}\right) \cdot p\left(s_{t} \mid x_{1}^{t-1}, s_{1}^{t-1}, w_{1}^{N}\right) \\
& =\sum_{s_{1}^{T}} \prod_{t=1}^{T} p\left(x_{t} \mid s_{t}, w_{1}^{N}\right) \cdot p\left(s_{t} \mid s_{t-1}, w_{1}^{N}\right)
\end{aligned}
$$

where the sum in Eq. (5) expresses all viable paths that lead to the same output sequence $w_{1}^{N}$. Equations (6) and (7) constitute reformulations with help of the chain rule. Assuming $s$ to be non-observable and a first order Markov process leads to Eq. (8). After applying the viterbi approximation, which considers only the most likely path and substituting everything into Eq. (4), we get:

$$
\begin{aligned}
{\left[w_{1}^{N}\right]_{\mathrm{opt}}=} & \underset{w_{1}^{N}}{\operatorname{argmax}}\left\{p ( w _ { 1 } ^ { N } ) \cdot \operatorname { m a x } _ { s _ { 1 } ^ { T } } \left\{\prod_{t=1}^{T} p\left(x_{t} \mid, s_{t}, w_{1}^{N}\right)\right.\right. \\
& \left.\left.\cdot p\left(s_{t} \mid s_{t-1}, w_{1}^{N}\right)\right\}\right\}
\end{aligned}
$$

where in the legacy Gaussian mixture model (GMM)-hidden Markov model (HMM) approach for sign language recognition $p\left(x_{t} \mid, s_{t}, w_{1}^{N}\right)$ has typically been modelled as

$$
\begin{aligned}
p\left(x_{t} \mid, s_{t}, w_{1}^{N}\right) & =\sum_{m=1}^{M} c_{m} \cdot \mathscr{N}\left(x, \mu_{m}, \Sigma\right) \\
\sum_{m=1}^{M} c_{m} & =1
\end{aligned}
$$

where $\mathscr{N}(x, \mu, \Sigma)$ is a multi-variate Gaussian with mean $\mu$, covariance matrix $\Sigma$ and $M$ is the number of mixture components (can differ between states of the same word). Legacy systems typically employed a globally pooled covariance matrix $\Sigma$ to cope with the low amount of training samples per state and word. The expectation maximization (EM) algorithm is used to estimate the sufficient statistics of the GMMs. The number of EM iterations is usually optimised on held out data during the training phase of the system. $p\left(s_{t} \mid s_{t-1}, w_{1}^{N}\right)$ (referring to Eq. (9)) represents the state transition model, which is empirically known as part of the model having limited impact on the final result and can therefore be pooled across all HMM states. In log-domain we often refer to it as the Time Distortion Penalties (TDPs). The dependency on the sequence of words $w_{1}^{N}$ may be dropped, since the temporal sequence of states $s_{1}^{T}$ is defined to be a sequence of HMM states corresponding to a specific path through the word sequence $w_{1}^{N}$, which we implement as concatenation of automatons for $w_{1}^{N}$ (using the word-to-state decomposition defined by the pronunciation lexicon and the word sequence annotations of the corpus).

\subsection{Hybrid CNN-HMM Approach}

Up to this point, we have deduced the standard HMM formula for recognition using a generative model for the emission probability. However, in the scope of the presented work we model the emission probability of the HMM $p\left(x_{t} \mid s_{t}, w_{1}^{N}\right)$ by an embedded $\mathrm{CNN}$, which is known to possess much more powerful image modelling capabilities than generative models such as GMMs. However, as pointed out by Richard and Lippmann (1991) and Bourlard and Morgan (1993), the $\mathrm{CNN}$ is a discriminative model whose outputs are estimates of the posterior probability and therefore cannot directly be inserted in the optimisation formula. Inspired by the hybrid approach known from ASR (Bourlard and Morgan 1993), we use Bayes' rule to convert the posterior probability of the $\mathrm{CNN}$ to a likelihood. For easier understanding we introduce the sub-word label $\alpha:=s, w_{1}^{N}$, representing the state $s$ belonging to the word sequence $w_{1}^{N}$. The CNN will hence be trained to model $p\left(\alpha \mid x_{t}\right)$. We apply Bayesian inference, converting the posteriors to class-conditional likelihoods following Bayes' rule:

$p\left(x_{t} \mid \alpha\right)=p\left(x_{t}\right) \cdot \frac{p\left(\alpha \mid x_{t}\right)}{p(\alpha)}$

where the prior probability $p(\alpha)$ can be approximated by the relative state label frequencies in the frame-state-alignment used to train the CNN.

For practical usage, we add several hyper-parameters to the implementation. These allow us to control the effect of the language model $(\gamma)$ and the $\mathrm{CNN}$ label prior $(\beta)$. Neglecting the constant frame prior $p\left(x_{t}\right)$, we finally optimise the following equation to find the best output sequence:

$$
\begin{aligned}
& {\left[w_{1}^{N}\right]_{\mathrm{opt}}} \\
& \quad=\underset{w}{\operatorname{argmax}}\left\{p\left(w_{1}^{N}\right)^{\gamma} \cdot \max _{s_{1}^{T}}\left\{\prod_{t=1}^{T} \frac{p\left(\alpha \mid x_{t}\right)}{p(\alpha)^{\beta}} \cdot p\left(s_{t} \mid s_{t-1}, w_{1}^{N}\right)\right\}\right\}
\end{aligned}
$$




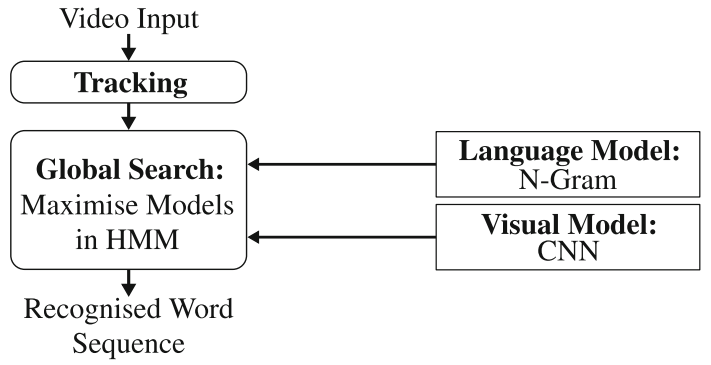

Fig. 1 Overview of the proposed CNN-HMM hybrid approach for continuous sign language recognition

A general overview of the proposed hybrid CNN-HMM algorithm for recognition can be found in Fig. 1. The hybrid approach has the positive property that during training only the $\mathrm{CNN}$ and the language model (LM) need to be retrained, while the HMM requires no training. For testing, the best hyper parameter values for $\gamma, \beta$ and the pooled state transition model $p\left(s_{t} \mid s_{t-1}, w_{1}^{N}\right)$ are found using a grid search.

Figure 2 summarises the resources we need to successfully apply the hybrid approach: a dual corpus of sign videos (sentence-wise segmented) and corresponding signword annotations. In this work, we further employ the HMM frame-state-alignment coming from a HMM-GMM system as frame labelling, which can be replaced by an appropriate re-alignment scheme as shown in Koller et al. (2017).

\subsection{Tandem Approach}

An intermediate step between GMM-HMM and the hybrid CNN-HMM systems is the so-called tandem approach. It is very similar to the hybrid approach in the sense that it uses both a CNN and HMM. However, the CNN is not used as a classifier, but rather as a feature extractor. In the so-called tandem approach (Hermansky et al. 2000) the activations of a fully connected layer or the feature maps of a convolutional layer are dumped, post-processed (Koller et al. 2016a) and then modelled in a GMM-HMM framework. This creates a significantly higher computational cost than the hybrid approach for extracting features and retraining a GMM system. Golik et al. (2013) found that in speech and handwriting

\section{Provided Training Ressources: $\longrightarrow$ Resulting Models:}

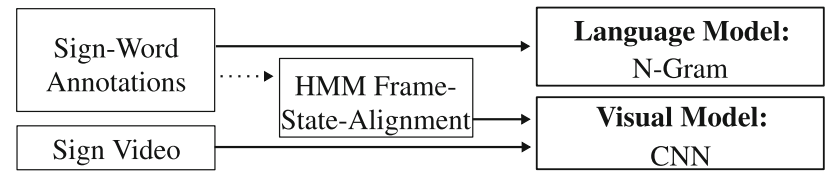

Fig. 2 Showing the employed resources (in light boxes on the left) to train the models for the hybrid CNN-HMM approach. The frame-statealignment has been generated from the sign-word (gloss-) annotations using a GMM-HMM system from Koller et al. (2016a) recognition the hybrid approach shows equal or superior performance compared to the tandem approach. We will verify this statement for sign language recognition in Sect. 6.3. As discussed in Sect. 2, in the gesture and sign language recognition literature to date, most other works either use the CNN outputs not in a Bayesian interpretation (Wu et al. 2016) or employ the CNN as feature extractor comparable to the tandem approach. Figure 3 shows the tandem and the hybrid approach side by side. We denote that the only difference is the visual model.

\section{Data Sets}

The experiments are carried out on three state-of-theart continuous sign language data sets that have been used extensively to compare recent methods for continuous sign language recognition: RWTH-PHOENIX-Weather 2012, RWTH-PHOENIX-Weather 2014 and SIGNUM. Here, we provide an essential summary and some additional statistics on the word-class distributions. However, for further details on the data sets, the interested reader is directed to Koller et al. (2015a).

Single images of the corpora are depicted in Fig. 4.

Brief statistics on the three data sets can be found in Table 1 . Both RWTH-PHOENIX-Weather corpora (2012 and 2014) were first introduced by Forster et al. (2012) and Forster et al. (2014) and represent direct recordings of the broadcast news, being limited to the weather forecast domain. As such, the data can be regarded as challenging real-life footage covering most difficulties you would expect from natural data (motion blur, transmission artifacts, fast signing, incomplete sentences, mis-signed words, interpretation errors, different clothing, etc.). RWTH-PHOENIX-Weather 2012 features a single signer interpreting the news into sign language, while RWTH-PHOENIX-Weather 2014 contains nine individuals covering varying amounts of the recorded programs.

SIGNUM was first introduced by von Agris et al. (2008b) and was recorded in a laboratory environment while carefully controlling the signing and recording conditions. However, deviations from word counts in Table 1 w.r.t. previous work are errata, while the underlying data has not changed. All data sets feature user-dependent setups as all individuals occur both in the training and in the test/development (dev) partitions. The RWTH-PHOENIX-Weather is freely available. ${ }^{1}$ It has to be noted that the actual annotation of PHOENIX 2014 and SIGNUM cover a larger variety of words than what the actual testing regime foresees. Therefore both data sets provide some mapping in order to join certain classes. This mainly arises due to the difficulty of the gloss annotation

\footnotetext{
${ }^{1}$ It can be obtained at http://www-i6.informatik.rwth-aachen.de/ koller/RWTH-PHOENIX/.
} 


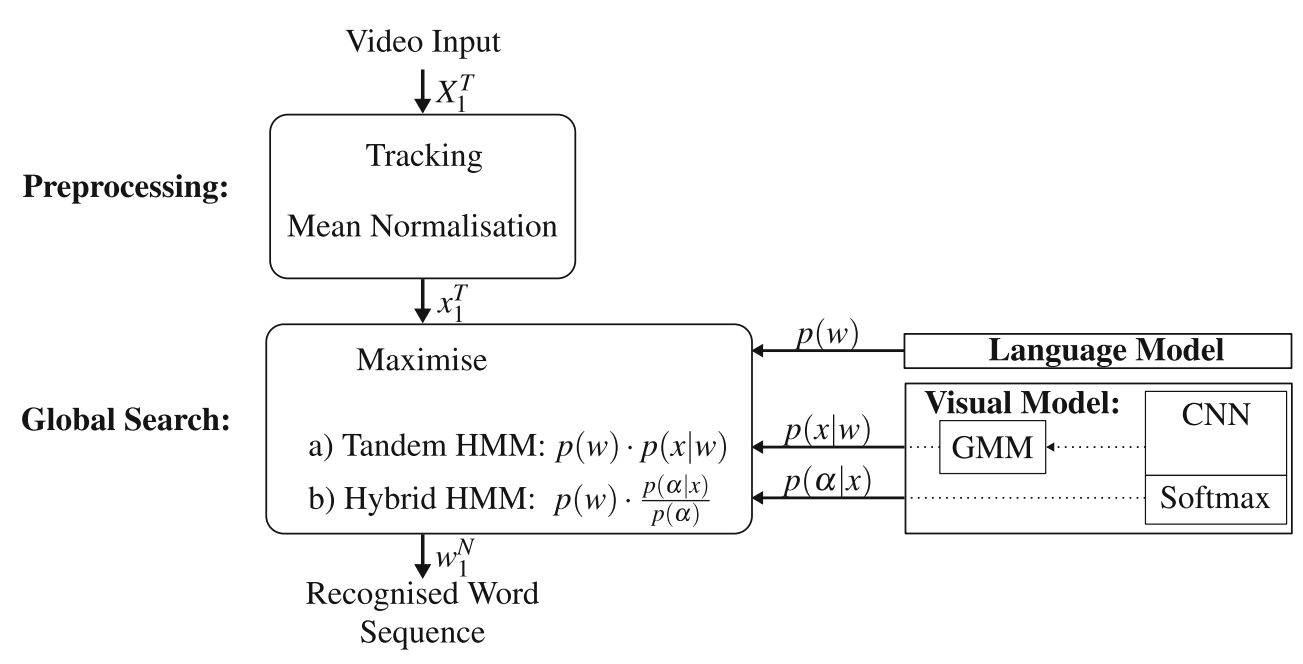

Fig. 3 Illustrating the difference between CNN-HMM tandem and hybrid approach. The former uses the CNN only as a feature extractor to train a subsequent GMM, while the later directly uses the CNN's normalised posteriors probabilities for a label $\alpha$ given input $x$

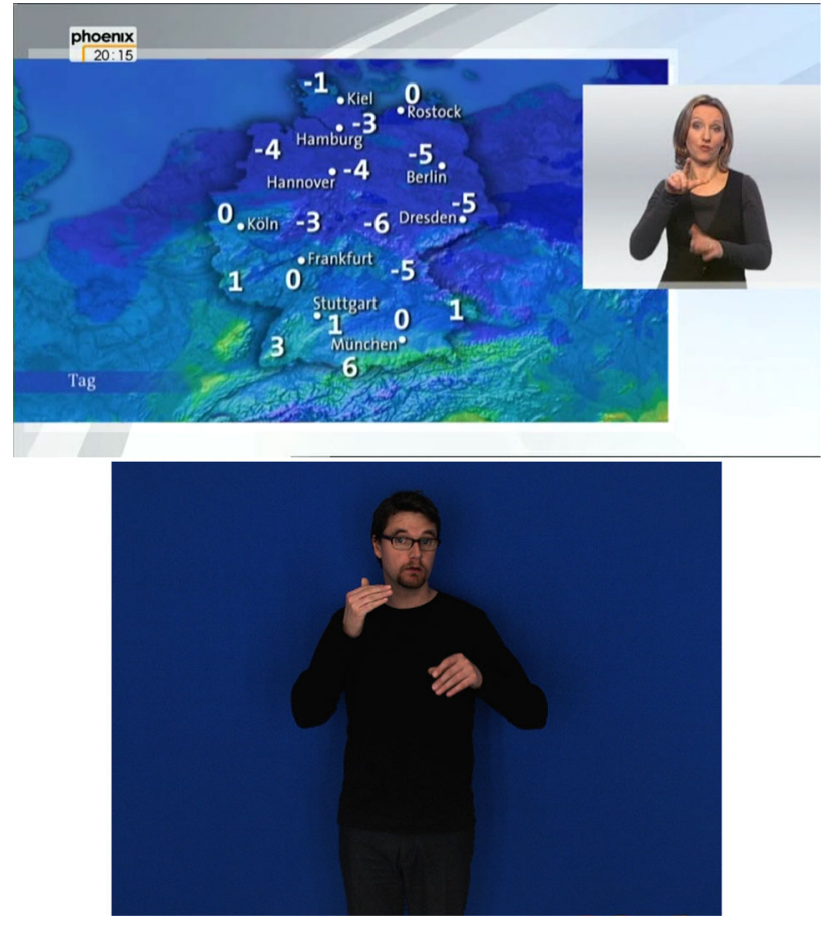

Fig. 4 Example images showing the data sets employed in this work. RWTH-PHOENIX-Weather on the left and SIGNUM on the right

and manifests itself partly in inflected forms of the same words and in different words that are visually identical or very close. All referenced publications that report results on the data sets have been applying this simplification scheme, which is distributed with the data. The final number of classes that are distinguished in evaluation (see row 'vocabulary' in Table 1) is 266, 1080 and 465 for PHOENIX 2012, PHOENIX 2014 and SIGNUM respectively. On SIGNUM the vocabulary is 10 words larger than the reported vocabu- lary by the authors (von Agris et al. 2008a). It is unclear what the cause for this is. Unfortunately the original authors cannot be reached anymore. The still frames in Table 1 refer to frames that have been automatically labelled as background during the HMM alignment.

Figures 5, 6 and 7 show the distribution of word counts on PHOENIX 2012, PHOENIX 2014 and SIGNUM respectively. It can be seen that both PHOENIX 2012 and 2014 contain a large number of words with only a single occurrence during training (so-called singletons), while SIGNUM statistics are different. On SIGNUM even the least frequent words occur at least 3 times, while most of them can be found at least 10 times in the training data. This is good for training and demonstrates SIGNUM's artificial characteristic which (among other reasons) manifests itself in very low WERs.

\section{Implementation Details}

In this section, we describe the details to allow exact reproducibility of our experiments. Note, that we input single (still) frames to the CNN and the HMM covers the temporal modeling. Input frames are cropped hand images. The system has no explicit information on the location other than from the background of the cropped images.

\subsection{Image Preprocessing}

To track the right hand across all sequences of images we use a dynamic programming based approach (Dreuw et al. 2006). In all data sets the right hand corresponds to the signer's dominant hand, which is the hand that plays the principle role in signing. On the RWTH-PHOENIX-Weather corpora, we crop a rectangle of $92 \times 132$ pixel around the centre of the 
Table 1 Key statistics of the employed data sets

\begin{tabular}{|c|c|c|c|c|c|c|c|}
\hline & \multicolumn{2}{|c|}{ PHOENIX 2012} & \multicolumn{3}{|c|}{ PHOENIX 2014} & \multicolumn{2}{|c|}{ SIGNUM } \\
\hline & Train & Test & Train & Dev & Test & Train & Test \\
\hline \# Signers & 1 & 1 & 9 & 9 & 9 & 1 & 1 \\
\hline Hours & 0.51 & 0.07 & 8.88 & 0.84 & 0.99 & 3.86 & 1.06 \\
\hline Frames & 46282 & 6751 & 799006 & 75186 & 89472 & 416620 & 114230 \\
\hline$\sim$ Still frames & - & - & $20 \%$ & - & - & $38 \%$ & - \\
\hline Running words & 3309 & 487 & 65227 & 5540 & 6504 & 11127 & 2805 \\
\hline$\varnothing$ Frames/word & 14.0 & - & 9.8 & - & - & 23.2 & \\
\hline Vocabulary & 266 & - & 1080 & - & - & 465 & - \\
\hline OOVs running & - & 8 & - & 28 & 35 & - & 9 \\
\hline OOVs [\%] & - & 1.6 & - & 0.5 & 0.5 & - & 0.3 \\
\hline
\end{tabular}

OOV Out-Of-Vocabulary, e.g. words that occur in test, but not in train. Dev refers to the development set

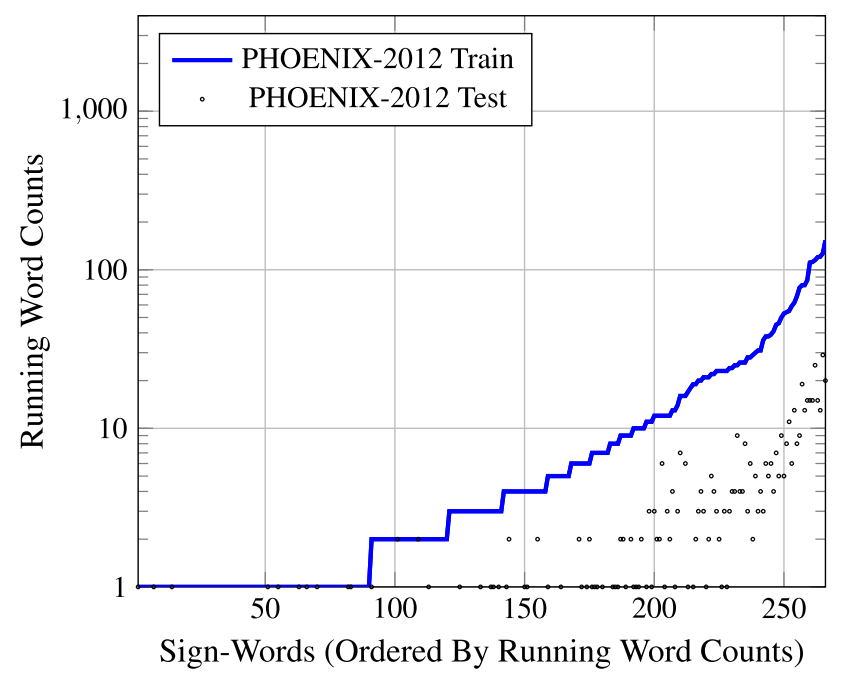

Fig. 5 Showing the distribution of words (and their counts) on the train and test partition of the RWTH-PHOENIX-Weather 2012 corpus. It can be seen that there are less than 100 sign-words occurring just a single time (singletons) imposing difficulties on the task

hand. The original images suffer a constant distortion due to the broadcast nature of the videos, which corresponds to a scaling of the image width by a factor of 0.7 . To compensate for this distortion we enlarge the cropped rectangles to the square size of $256 \times 256$. On SIGNUM we directly crop a square patch of size $100 \times 100$ pixel and scale it up to $256 \times 256$. Thereafter the pixel-wise mean of all images in the training set is subtracted from each image. Finally, for data augmentation we follow an online cropping scheme, which randomly crop out a $224 \times 224$ (GoogLeNet) or a $227 \times 227$ pixel (LeNet and AlexNet) rectangle to match the size of images in our model which was pre-trained on ImageNet. The input to the CNNs consists of single cropped hand patches.

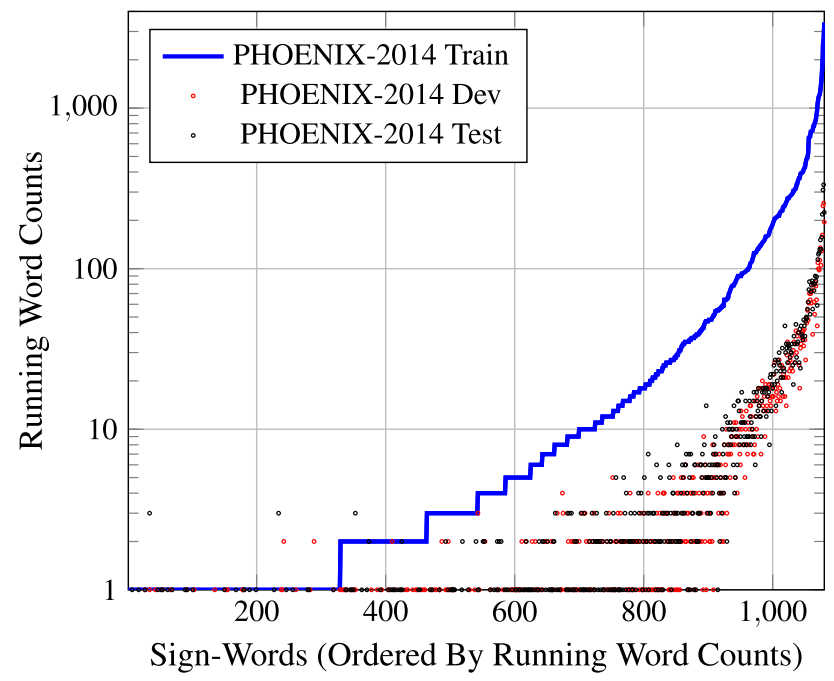

Fig. 6 Showing the distribution of words (and their counts) on the train, dev and test partition of the RWTH-PHOENIX-Weather 2014 corpus. It can be seen that there are more than 300 sign-words occurring just a single time (singletons) while few other classes occur more than 1000 times imposing difficulties on the task. Dev refers to the development set

\subsection{Convolutional Neural Network Training}

We base our CNN implementation on Jia et al. (2014), which uses the NVIDIA CUDA Deep Neural Network GPUaccelerated library. If not stated otherwise in the respective experiments, we opted for the GoogLeNet Szegedy et al. (2015) 22 layers deep CNN architecture with around 15 million parameters (for exact parameters refer to Table 3). GoogLeNet has shown many times in the past, most notably in the ImageNet 2014 (ILSVRC) Challenge, that it can be quite effective in combining impressive performance with minimal computational resources. Much of the improvements in this architecture compared to others' stems from the inception module which combines filters of different sizes after applying dimensionality reduction through a $1 \times 1$ Con- 


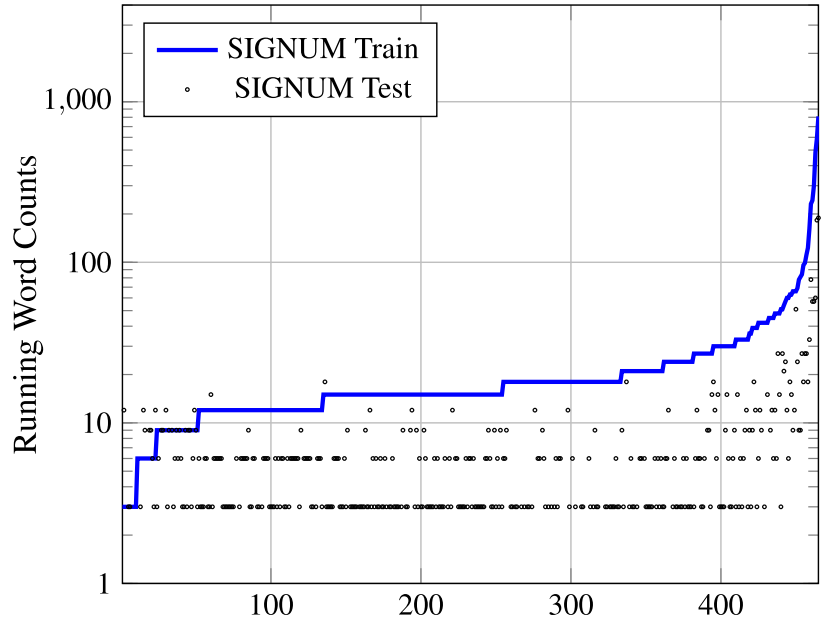

Sign-Words (Ordered By Running Word Counts)

Fig.7 Showing the distribution of words (and their counts) on the train, dev and test partition of the SIGNUM single signer corpus. In large contrast to the RWTH-PHOENIX-Weather corpora, it can be seen that hardly any sign-words occur just a single time (singletons). This shows the artificial nature of the data set and explains its comparative easiness

\section{Train (legacy) GMM-HMM}
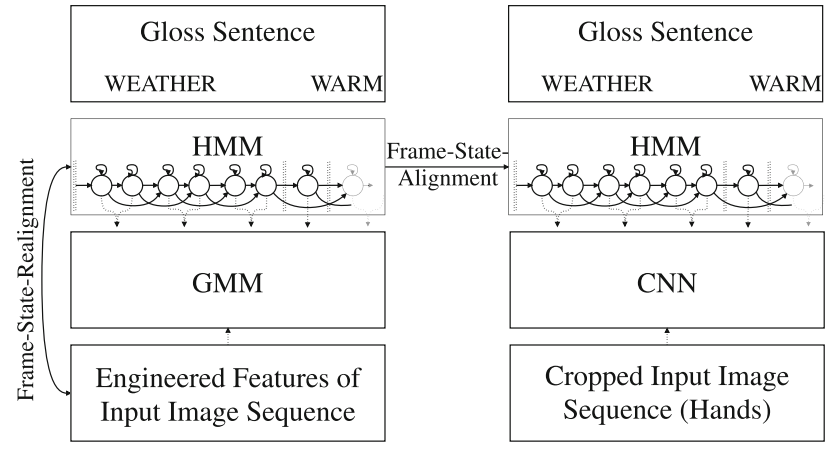

Fig. 8 Illustration of training scheme

volutional layer. The employed $\mathrm{CNN}$ architecture includes 3 classifying layers, meaning that besides the final classifier the network also includes two intermediary auxiliary classifiers. Those encourage discrimination in earlier layers of the network. The loss of these auxiliary classifiers is added to the total loss with a weight of 0.3 . All non-linearities are rectified linear units and each classifier layer is preceded by a dropout layer. We use a dropout rate of 0.7 for the auxiliary layers and 0.4 before the final classifier.

As mentioned in Sect. 3, the CNN training scheme requires an initial frame-state-alignment. This originates from a GMM-HMM recognition system that is trained to re-aligning the frame-to-state mapping (frame-level alignment). This is illustrated in Fig. 8. If not stated otherwise in the respective experiments, we use alignments from GMM-HMM systems reproducing the best published results on our chosen corpora.
For SIGNUM and RWTH-PHOENIX-Weather 2014 we use the best results published in Koller et al. (2016a) as alignment, whereas for RWTH-PHOENIX-Weather 2012 we use Koller et al. (2015a). We split the frame-level alignment into a training ( $\sim 90 \%$ of the data) and a validation set $(\sim 10 \%$ of the data) in order to be able to evaluate the per-frame accuracy of the CNN and stop the training when the validation accuracy deteriorates. However, we noticed that this seldom happened and in these experiments we always chose the last training iteration. We first train the network on the ImageNet data set with 1.2 million high-resolution images in 1000 classes and then exchange the final classification layers (on all three classifiers) and finetune the network on the sign language data for 80,000 iterations with a mini-batch size of 32 images. We use stochastic gradient descent with an initial learning rate $\lambda_{0}=0.01$ for CNN networks. We employ a polynomial scheme to decrease the learning rate $\lambda_{i}$ for iteration $i$ as the training advances while reaching $\lambda_{i}=0$ for the maximum number of iterations $i_{\max }=80 \mathrm{k}$ in our experiments for 4 epochs on PHOENIX (2012 and 2014) and SIGNUM. Only the experiment in Sect. 6.4 that analyses the effect of the HMM structure does not use the training and validation splitting. Instead it uses all available training data for training the CNN. Therefore we train for 100k iterations here.

$\lambda_{i}=\lambda_{0} \cdot\left(1-\frac{i}{i_{\max }}\right)^{0.5}$

\subsection{CNN Inference}

Once the CNN training is finished, we consider all three classifiers (the main one and the two auxiliary ones) for estimating the best performing iteration. For the proposed hybrid CNN-HMM approach we add a softmax and use the resulting posteriors in our HMM as observation probabilities.

In the tandem CNN-HMM approach we employ the activations from the last layer before the softmax that yields the highest accuracy on the validation data. With RWTHPHOENIX-Weather 2012, this is a fully connected layer of the first auxiliary classifier, possibly because the data set does not provide enough data for training an earlier softmax. For RWTH-PHOENIX-Weather 2014 and SIGNUM the pooling layer before the main classifier yields 1024 values. The tandem system requires feature extraction for both training and test sets, since a GMM-HMM system is retrained with them. After a global variance normalisation, we apply PCA to reduce the feature dimension to 200 .

\subsection{Continuous Sign Language Recognition}

We base the HMM part of this work on the freely available state-of-the-art open source speech recognition system 
RASR Rybach et al. (2011). Following the hybrid approach we use the posterior probabilities from the $\mathrm{CNN}$, as well as the corresponding class priors. In the following experiments the prior-scaling-factor $\beta$ is set to 0.3 if not stated otherwise. The LM is estimated as n-gram using the SRILM toolkit by Stolcke (2002). The HMM is employed in bakis structure (Bakis 1976). This is a standard left-to-right structure with forwards, loops and skips across at most one state. Additionally, two subsequent states share the same class probabilities. The transition model $p\left(s_{t} \mid s_{t-1}, w_{1}^{N}\right)$ is pooled across all sign-words. As we actually perform the search in $\log$ space we call the transition model TDPs. The TDPs define the transition penalties that account for state changes in the HMM. The garbage class is modelled as an ergodic state with separate transition penalties to add flexibility, such that it can always be inserted between sequences of sign-words. As for RWTH-PHOENIX-Weather 2014 and SIGNUM, we model each sign-word with three hidden states. However, in RWTH-PHOENIX-Weather 2012 we employ a length modelling scheme where sign-words are represented by more or fewer states depending on their average alignment length. For details on the employed length modelling consult Koller et al. (2015a). In agreement to most sign language recognition literature, we measure the system performance in WER. WER is based on the Levenshtein alignment between reference and hypothesis sentence and it measures the required numbers of deletion, insertion and substitution operations to transform the recognised hypothesis into the reference sequence.

$\mathrm{WER}=\frac{\text { \#deletions }+ \text { \#insertions }+ \text { \#substitutions }}{\text { \#reference observations }}$

For recognition, we perform a grid search over possible hyper parameters for $\gamma, \beta$ and the TDPs. As such, the forward, loop, skip and exit transition penalties are optimised on the dev set (or if not available on the test set) in order to minimise the WER. RASR provides an efficient implementation of the word conditioned tree search which is based on the concepts described in Ney and Ortmanns (2000), which is used for this work. In brief, for each time step the search expands all possible state hypotheses and maintains them in memory. The current score of a hypothesis is composed of the visual score $-\log \left(\frac{p\left(x_{t} \mid \alpha\right)}{p(\alpha)^{\beta}}\right)$ and the transition penalty $-\log \left(p\left(s_{t} \mid s_{t-1}\right)\right)$. Whenever a sign-word ends (which manifests itself in leaving the last state of the HMM), the language model score $-\log \left(p(w)^{\gamma}\right)$ and the exit penalty are also added (refer to Sect. 3, specifically Eq. (13) for the exact composition of the search formula). The maximum-approximation (cfSect. 3) allows recombination of state hypotheses that have reached the same state at the same time with the same sign-word history. This significantly limits the combinatorial explosion of the number of search hypotheses. Furthermore, the search space is pruned in order to boost performance and reduce memory consumption. We perform histogram and threshold pruning. The latter acts like a beam search. At each time step, only sign-word hypotheses with scores relatively close to the best hypothesis are allowed. All others are discontinued and therefore removed from memory. This maximum distance from the best hypothesis is represented by the visual threshold pruning value (in $\log$ domain). After adding the language model score at the word end the LM threshold pruning is applied in the same way. The histogram pruning uses a histogram to limit the amount of hypotheses to the given value. The visual histogram pruning is applied at every state, whereas the LM histogram pruning is only applied after the language model score has been added to each hypothesis at sign-word end states. Table 2 summarises the respective pruning settings for each of the data sets.The exact hyper parameter values for the transition probabilities are given for each experimental description, as they vary from experiment to experiment.

\subsection{Computational Requirements}

Using a GeForce GTX 980 GPU with 4GB memory, training on the PHOENIX 2012 data set is done at the speed of $\sim 150$ frames per second (fps) and inference at a rate of $\sim 450 \mathrm{fps}$. Using the same hardware on PHOENIX 2014 data set yields $\sim 35$ fps for training and $\sim 350$ fps for inference. SIGNUM runs at $\sim 10 \mathrm{fps}$ during training and $\sim 56 \mathrm{fps}$ for inference. HMM recognition is done at $\sim 2 \mathrm{fps}$ for PHOENIX 2012 and due to the tighter pruning $\sim 25 \mathrm{fps}$ for PHOENIX 2014, while SIGNUM runs at $\sim 8$. The HMM parameter optimisation took a total of $\sim 38 \mathrm{~h}$ for PHOENIX 2012, $\sim 130 \mathrm{~h}$ for PHOENIX 2014 and $\sim 65 \mathrm{~h}$ for SIGNUM using a single core machine with 2GB RAM.

The training and recognition pipelines have not been optimised for speed. We load individual image files from a file server, which acts as a significant bottleneck. We have experimented with a leveldb database, which is able to double the speed roughly.

\section{Experiments}

In this section we present experimental evaluation to help estimate key factors influencing the performance of a $\mathrm{CNN}$ HMM hybrid system on the task of sign language recognition. In the next subsection we first analyse the effect of the $\mathrm{CNN}$ structure on the final recognition performance. Then, in Sect. 6.2 we evaluate the effect of additional out-of-domain training data. In Sect. 6.3 we compare the hybrid and the tandem approach, before we analyse the effect of the HMM structure in Sect. 6.4. In Sect. 6.6 we provide a general overview comparison against the state-of-the-art, while in Sect. 6.5 we assess model ensembles. 
Table 2 Showing the pruning values for each of the data sets

\begin{tabular}{llrr}
\hline Type of pruning & PHOENIX 2012 & PHOENIX 2014 & SIGNUM \\
\hline Visual threshold & None & 2000 & 2000 \\
Visual histogram & None & 20,000 & 20,000 \\
LM threshold & None & 4000 & 4000 \\
LM histogram & None & 10,000 & 10,000 \\
\hline
\end{tabular}

No pruning is necessary with PHOENIX 2012

Table 3 Showing number of parameters (weights+biases) in millions of different CNN structures adapted to our tasks: PHOENIX 2012:1443 outputs PHOENIX 2014: 3694 outputs SIGNUM: 1366 outputs

\begin{tabular}{|c|c|c|c|c|c|c|c|c|}
\hline \multirow[t]{2}{*}{ NN-structure } & \multirow[t]{2}{*}{ Input size $[\mathrm{px}]$} & \multirow[t]{2}{*}{ \#layers } & \multicolumn{2}{|c|}{ PHOENIX 2012} & \multicolumn{2}{|c|}{ PHOENIX 2014} & \multicolumn{2}{|c|}{ SIGNUM } \\
\hline & & & \multicolumn{2}{|c|}{ \#params (last fc) $\left[10^{6}\right]$} & \multicolumn{2}{|c|}{ \#params (last fc) $\left[10^{6}\right]$} & \multicolumn{2}{|c|}{ \#params (last fc) $\left[10^{6}\right]$} \\
\hline LeNet & $227 \times 227$ & 4 & 73.6 & $(0.7)$ & 74.7 & (1.8) & 73.6 & $(0.6)$ \\
\hline AlexNet & $227 \times 227$ & 8 & 62.7 & (5.9) & 72.0 & $(15.1)$ & 62.4 & $(5.5)$ \\
\hline GoogLeNet & $224 \times 224$ & 22 & 14.7 & (1.4) & 21.6 & (3.7) & 14.5 & (1.4) \\
\hline
\end{tabular}

\subsection{Effect of CNN Structure}

A crucial research question is to estimate the effect of the $\mathrm{CNN}$ architecture on a specific task. This subsection aims to provide an answer to this question by applying different CNN structures to the task of sign language recognition, while all remaining hyper parameters are fixed (we adjust the transition probabilities for each experiment). As such, we compare three well-known CNN architectures. All three have, at some point in time, received much attention by the community for outperforming the state-of-the-art largely on different classification tasks. LeNet (full name is LeNet-5), introduced by LeCun et al. (1998), was the first successful CNN having 4 non-linear layers. Its application was character recognition of the MNIST digits (LeCun et al. 1998) with a size of $32 \times 32$ pixel. In this work, we employ a version which deviates from the original implementation by the number and kind of non-linearities. For simplicity we chose the version distributed jointly with the caffe framework (Jia et al. 2014). The other two popular architectures analysed in the scope of this work were winners of the ImageNet Large Scale Visual Recognition Challenge (ILSVRC) in 2012 an 2014. AlexNet by Krizhevsky et al. (2012) was the first deeper CNN with 8 layers (5 convolutional and 3 fully connected layers). It won the object classification competition with a top-5 error of $15.4 \%$ across the targeted 1000 classes. Two years later Szegedy et al. (2015) won the competition with GoogLeNet. A 22-layer deep CNN that achieved a top-5 error of $6.67 \%$ on the task. In order to facilitate the comparison of the three mentioned architectures, we have compiled their key characteristics in Table 3. Note that the number of parameters for GoogLeNet includes the parameters used for the two additional auxiliary softmax classifiers. Table 3 shows the input size, the number of non-linear layers and the number of parameters of the whole network (whose last output layers have been adjusted to each of the three data sets analysed in this work). It also shows the number of parameters of the last fully connected classification layer, which often makes up the largest part in the network and varies from task to task. The last layer's size is due to the large amount of sign-labels $\alpha$ (cfSect. 3 for details). $\alpha$ represents the labels belonging to the three hidden states that model each of the sign classes (over 1000) from our vocabulary (for PHOENIX 2014).

Discussion of Results Table 4 summarises the experimental results comparing the different $\mathrm{CNN}$ architectures. We see that GoogleNet clearly outperforms the other architectures on both tasks with at least $4 \%$ relative improvement in WER. We further see that it is clearly not just the number of parameters that determines the model quality but rather the number of non-linear layers.

\subsection{Effect of Finetuning}

In this experiment we want to evaluate the effect of using out-of-domain data to train the networks prior to finetuning them on the actual in-domain task using specific but quite limited training data. We therefore make use of the 1.2 million labelled images from the ILSVRC to train the networks. After that we exchange the final fully-connected classification layer and fine-tune the network. In case of the GoogLeNet architecture we exchange the layers of both auxiliary classifiers as well. We perform the experiment with the AlexNet and the GoogLeNet architectures.

Discussion of results Tables 5 and 6 report the results for the AlexNet and GoogLeNet architecture, respectively. For both architectures out-of-domain training and subsequent 
Table 4 Comparing different CNN structures

Table 5 Comparing the effect of pretraining $\mathrm{CNN}$ structures on out-of-task data: ILSVRC 2014

Table 6 Comparing the effect of pretraining $\mathrm{CNN}$ structures on out-of-task data: ILSVRC 2014

\begin{tabular}{|c|c|c|c|c|}
\hline \multirow[t]{2}{*}{ CNN Structure } & \multirow{2}{*}{$\frac{\text { PHOENIX } 2012}{\text { Test }}$} & \multicolumn{2}{|c|}{ PHOENIX 2014} & \multirow{2}{*}{$\begin{array}{l}\text { SIGNUM } \\
\text { Test }\end{array}$} \\
\hline & & Dev & Test & \\
\hline LeNet $(227 \times 227$ input $)$ & 47.8 & 69.5 & 68.4 & 17.9 \\
\hline AlexNet & 51.5 & 45.5 & 44.5 & 10.6 \\
\hline GoogLeNet & 34.1 & 43.1 & 42.7 & 8.9 \\
\hline
\end{tabular}

Results in WER [\%]: the lower the better

\begin{tabular}{|c|c|c|c|c|}
\hline \multirow[t]{2}{*}{ AlexNet } & \multirow{2}{*}{$\frac{\text { PHOENIX } 2012}{\text { Test }}$} & \multicolumn{2}{|c|}{ PHOENIX 2014} & \multirow{2}{*}{$\begin{array}{l}\text { SIGNUM } \\
\text { Test }\end{array}$} \\
\hline & & Dev & Test & \\
\hline Randomly initialised & 51.5 & 45.5 & 44.5 & 10.6 \\
\hline Fine-tuned & 39.2 & 42.2 & 41.1 & 8.7 \\
\hline
\end{tabular}

The first line represents training from scratch using the AlexNet structure, whereas the second corresponds to finetuning weights learnt on Imagenet. Results in WER [\%]: the lower the better

\begin{tabular}{lllll}
\hline GoogLeNet & PHOENIX 2012 & \multicolumn{2}{l}{ PHOENIX 2014 } & SIGNUM \\
\cline { 2 - 5 } & Test & Dev & Test & Test \\
\hline Randomly initialised & 34.1 & 43.1 & 42.7 & 8.9 \\
Fine-tuned & 30.0 & 38.3 & 38.8 & 7.4 \\
\hline
\end{tabular}

The first line represents training from scratch using the GoogLeNet structure, whereas the second corresponds to finetuning weights learnt on Imagenet. Results in WER [\%]: the lower the better finetuning yields clear gains. With AlexNet we see $30 \%$ relative improvement on PHOENIX 2012, $8 \%$ on PHOENIX 2014 and $20 \%$ on SIGNUM, while with GoogLeNet we see $13 \%$ relative improvement on PHOENIX 2012, over 10\% on PHOENIX 2014 and again 20\% on SIGNUM. We conclude that strongly supervised out-of-domain data has a consistently positive influence on learning hybrid sign language models-at least if the out-of-domain data is as diverse as ImageNet.

\subsection{Hybrid Compared to Tandem Modelling}

In this subsection we want to explore the question of whether it is better to use the CNN's outputs as features and train a subsequent GMM-HMM system in the so-called tandem approach (Sect. 3.3) or to directly use the posteriors as observation probabilities as in the presented hybrid approach.

Discussion of results Figure 9 compares the hybrid CNNHMM modelling against the tandem modelling. We can see that the hybrid approach slightly outperforms the tandem approach on all three data sets. This is consistent with the literature as found by Golik et al. (2013) in speech and handwriting recognition. However, especially in terms of training complexity, the hybrid approach is clearly favourable as the subsequent GMM training is not necessary.

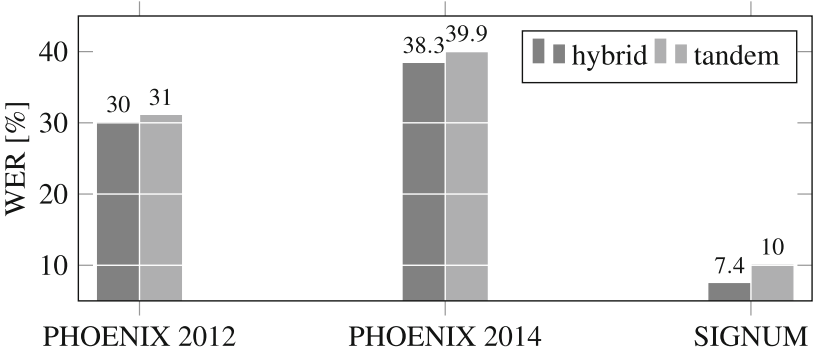

Fig. 9 The hybrid and the tandem approach side-by-side on all three data sets. Results in WER [\%]: the lower the better

\subsection{Effect of Hidden States}

Until this point, we have seen experiments estimating the effect of several components on the overall sign recognition pipeline. However, the question remains, how much the HMM impacts the final WER. It is clear that the HMM is the key element to allow the mapping from an input sequence of specific length to an output sequence of different length. But does the hidden state topology influence the final result in a similar way as the CNN structure or the CNN training?

In this subsection we analyse the effect of the HMM structure. More specifically, we want to know if multiple hidden states help the deep CNN to perform better or if they are a relic of the GMM-HMM architecture that strong CNNs make redundant. Therefore, we perform experiments on the 


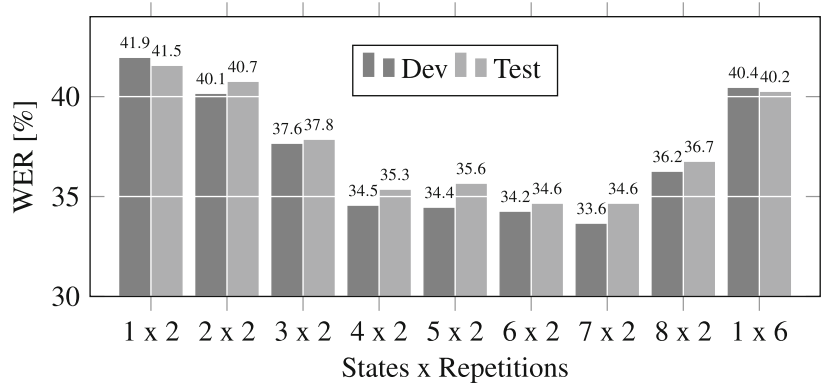

Fig. 10 Showing the best achieved WERs in [\%] (the lower the better) on PHOENIX 2014 for different numbers of states and repetitions

PHOENIX 2014 data set altering the HMM topology w.r.t. the number of hidden states. The baseline system corresponds to a HMM architecture that models each sign-word with 3 hidden states which are each repeated twice (sharing the same probabilities). Thus, this topology has 6 states, but only 3 probability distributions need to be estimated by the CNN. This standard bakis topology ensures that we can compensate for variation in signing speed by skipping states. By definition, we can skip at most one state. The repetitions therefore ensure that all emission probabilities have to be visited at least a single time. In order to allow for valid conclusions, we need to make sure that all systems have the chance to find a good alignment w.r.t. their HMM architecture. In opposition to all other experiments presented in the scope of this work, we therefore perform multiple iterations of re-alignment, where we re-estimate the viterbi path. We start from a flat segmentation, where the available frames are equally distributed across all states of a sentence. The re-alignment then iteratively updates the frame labelling and therefore affects the subsequent CNN training. Thus, after each re-alignment we perform a fine-tuning of the previous iteration's model for 100k iterations ( $\sim 4$ epochs). Each iteration takes about $6 \mathrm{~h}$ for CNN training and 20 minutes for viterbi alignment. We perform 10 re-alignment iterations for all different HMM topologies and report the best result among all iterations.

Discussion of results Figure 10 shows the results in terms of WER on the PHOENIX $2014 \mathrm{dev}$ and test partition. We first vary the amount of states per sign-word from 1 to 8 , maintaining the 2 state repetitions. In this setting, the baseline of 3 states and 2 repetitions clearly outperforms topologies with less states. However, we see the best performance further increasing the numbers of states to 7 . We note a WER difference between the weakest $(1 \times 2$ states $)$ and the strongest topology $(7 \times 2$ states $)$ of $8.5 \%$ absolute and over $20 \%$ relative. The 7 state architecture achieves $33.4 \%$ WER on the dev set and 34.4 on the test set. One could argue that it is the implied HMM length and not the division into hidden states that produces the improvements with longer HMMs. Therefore, we further perform an experiment with 1 state and 6 repetitions, which has the same length behaviour as the
Table 7 Showing how the HMM structure in terms of HMM states and repetitions affects the total number of HMM states and the neural network parameters (weights+biases) in millions

\begin{tabular}{lll}
\hline HMM structure & PHOENIX 2014 & \\
\cline { 2 - 3 } States $\times$ Repetitions & Total states & Parameters $\left[10^{6}\right]$ \\
\hline $1 \times 2$ & 1232 & 14.1 \\
$2 \times 2$ & 2463 & 17.9 \\
$3 \times 2$ & 3694 & 21.7 \\
$4 \times 2$ & 4925 & 25.4 \\
$5 \times 2$ & 6156 & 29.2 \\
$6 \times 2$ & 7387 & 33.0 \\
$7 \times 2$ & 8618 & 36.8 \\
$8 \times 2$ & 9849 & 40.6 \\
\hline
\end{tabular}

baseline. However, this model performs much worse than the baseline. As such, we can conclude that the HMM architecture has a strong influence on the recognition performance. Nevertheless, in Table 7 we can see how the number of HMM states affects the overall model size. This significantly impacts runtime.

\subsection{Effortless Ensemble of Models}

Finally, we want to show that a log-linear combination of multiple CNN models can further improve performance. We therefore define the probability by the visual model to be the combined product of each single model $i$ scaled by a factor $\delta_{i}$ as in

$p\left(x_{1}^{T} \mid w_{1}^{N}\right)=\max _{s_{1}^{T}}\left\{\prod_{t=1}^{T} \prod_{i}\left[\frac{p_{i}\left(\alpha \mid x_{t}\right)}{p_{i}(\alpha)^{\beta}}\right]^{\delta_{i}} \cdot p\left(s_{t} \mid s_{t-1}, w_{1}^{N}\right)\right\}$

In the scope of this work we combine $I=2$ models. The fact that model ensembles increase performance is well known. However, typically the building of models that are sufficiently complementary to yield any improvements constitutes a large computational overhead. In this section, we show that the process of re-aligning the models already adds sufficient discriminative information. Even models from successive re-alignment iterations yield strong gains when deployed as ensemble. This is remarkable as it means that with the proposed algorithm we get such models free of additional effort.

We choose two successive iterations of the best HMM architecture using 7 states and 2 repetitions, namely the 10th iteration yielding 33.6/34.6 and the 9th iteration yielding 33.8/34.6 on the development set and on the test set respectively. The log-linear combination with $\delta_{1}=0.87$ and $\delta_{2}=0.13$ yields a WER of $31.6 \%$ and $32.5 \%$ for dev and test respectively on PHOENIX 2014. This corresponds to a 


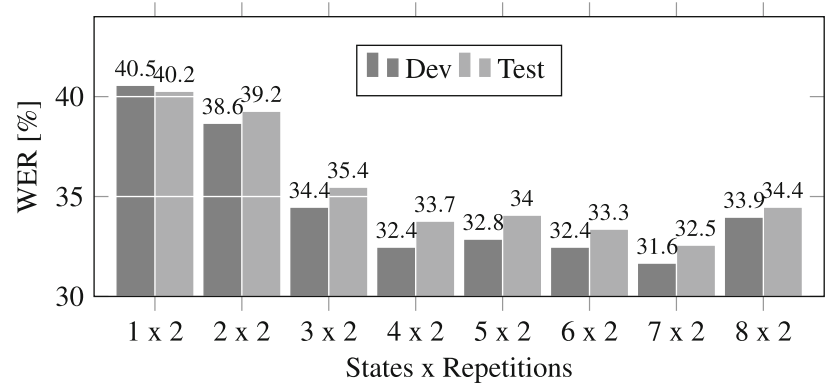

Fig. 11 Showing the best achieved WERs in [\%] (the lower the better) on PHOENIX 2014 for log-linear model combination of the two best alignment iterations (while keeping the HMM architecture fixed)

relative gain of around $6 \%$ compared to the single models (Fig. 11).

\subsection{General Comparison to State-of-the-Art}

Table 8 shows a detailed comparison to the state-of-the art on the three employed benchmark corpora. Besides performance measures, it reports the method of choice by the respective publications. Note that the proposed hybrid approach currently exploits only a single cropped hand of the signer and yet achieves state-of-the-art performance. Sign language is highly multimodal and makes heavy use of manual components (hand shape, orientation, place of articulation, movement) and also non-manual components (facial expression, eyebrow height, mouth, head orientation, upper body orientation). Most of the competing approaches use these additional modalities in recognition, which is why we expect additional gain when including them in the proposed approach. The previously best hand only result mentioned in Koller et al. (2016a) also relied on CNN models, but did not employ the hybrid approach end-to-end in recognition, loosing some performance due to this. It set the benchmark on PHOENIX 2014 Multisigner to 51.6\% WER. However, our proposed CNN-HMM achieves a strong result of $33.6 \%$ and $34.6 \%$ on dev and test respectively with a single model and $31.6 \% / 32.5 \%$ with model combination. This corresponds to about $20 \%$ absolute WER or over $38 \%$ relative improvement. On the single signer corpus RWTHPHOENIX-Weather 2012 the proposed approach improved the best baseline from $35.5 \%$ to $30.0 \%$, still being a relative improvement of over $15 \%$. On SIGNUM we improve the best known word error rates from from $12.0 \%$ to $7.4 \%$. As can be seen in Table 8, our hand-only hybrid CNN-HMM even outperforms multimodal approaches.

Nevertheless, the need to include more modalities than just the right hand is revealed by looking at the recognition errors. Qualitative examination of the top confusions on PHOENIX 2014 made by the hybrid approach highlight confused pairs such as "SNOW" with "RAIN" or "SHOWER" with "RAIN". However, these signs share the same hand configurations, whereas only the mouth shape changes. Given the classification relies purely on the right hand, it is understandable that it cannot distinguish between these signs. The top 30 confusions all relate to this type of error.

\section{Conclusion and Future Work}

In this work, we introduced an end-to-end embedding of a $\mathrm{CNN}$ into a HMM, while interpreting the outputs of the CNN in a truly Bayesian framework and training the system as a hybrid CNN-HMM in an end-to-end fashion Most state-ofthe-art approaches in gesture and sign language modelling use a sliding window approach or simply evaluate the output in terms of overlap with the ground truth. While this is sufficient for data sets that provide such training and evaluation characteristics, it is unsuitable for real world use. For the field to move forward more realistic scenarios, such as those imposed by challenging real-life sign language corpora, are required.

In this manuscript, we presented a hybrid CNN-HMM framework that combines the strong discriminative abilities of CNNs with the sequence modelling capabilities of HMMs, while abiding to Bayesian principles. This work represents the extended version of our previous work (Koller et al. 2016b), where we were the first to present such an embedding in the context of sign language and gesture recognition.

With the hybrid method we were able to achieve a large relative improvement of over $15 \%$ compared to the previous state-of-the-art on three challenging benchmark continuous sign language recognition data sets. On the two single signer data sets RWTH-PHOENIX-Weather 2012 and SIGNUM we improve the best known word error rates from 35.5 to $30.0 \%$ and from 12.0 to $7.4 \%$ respectively, while only employing basic hand-patches as input. On the difficult 9 signer $>1000$ vocab RWTH-PHOENIX-Weather 2014 Multisigner, we lower the error rates from $51.6 \% / 50.2 \%$ to $31.6 \% / 32.5 \%$ on dev/test.

In the scope of this extended manuscript, we significantly added to the theoretical explanation of the hybrid approach, with the aim of making its idea more accessible to newcomers to the field and presented much more extensive experiments: We analysed the effect of both CNN- and HMM-structure on the hybrid approach. We investigated the effect of using outof-domain data to train the network prior to finetuning using in-domain data. Finally, we showed that the use of ensembles of hybrid CNN-HMMs is able to further boost performance.

In terms of future work, we would like to extend our approach to cover all relevant modalities. Moreover, techniques to overcome the necessary initial alignment, such as end-to-end training will also be investigated. 
Table 8 Comparison with state-of-the-art

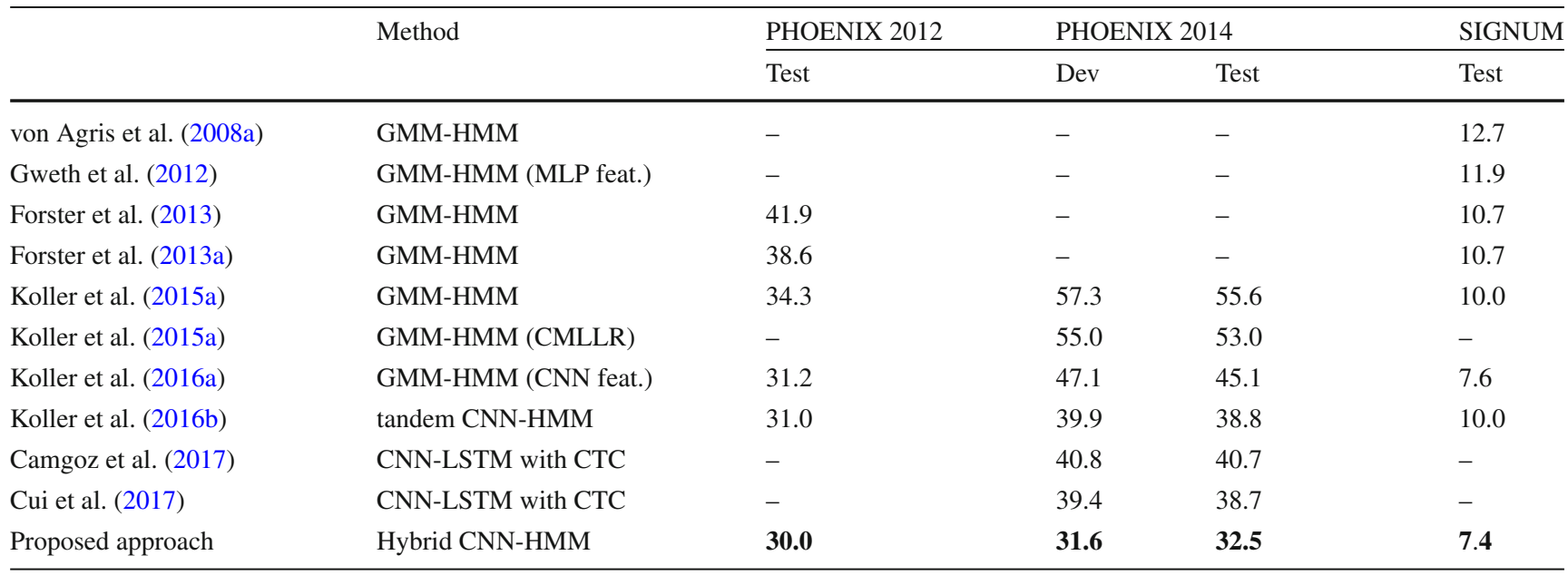

Best results are highlighted in bold

Results in WER [\%]: the lower the better. Best results of the proposed approach are single models. Model combination further improves the error on PHOENIX 2014 down to $34.4 \%$

Open Access This article is distributed under the terms of the Creative Commons Attribution 4.0 International License (http://creativecomm ons.org/licenses/by/4.0/), which permits unrestricted use, distribution, and reproduction in any medium, provided you give appropriate credit to the original author(s) and the source, provide a link to the Creative Commons license, and indicate if changes were made.

\section{References}

Assael Y. M., Shillingford, B., Whiteson, S., \& de Freitas, N. (2016). LipNet: End-to-end sentence-level lipreading. arXiv:1611.01599

Bahl, L. R., Jelinek, F., \& Mercer, R. L. (1983). A maximum likelihood approach to continuous speech recognition. IEEE Transactions on Pattern Analysis and Machine Intelligence, PAMI-5(2), 179-190. https://doi.org/10.1109/TPAMI.1983.4767370.

Bakis, R. (1976). Continuous speech recognition via centisecond acoustic states. The Journal of the Acoustical Society of America, 59(S1), S97-S97. https://doi.org/10.1121/1.2003011.

Bengio, Y., \& Frasconi, P. (1996). Input-output HMMs for sequence processing. IEEE Transactions on Neural Networks, 7(5), 12311249.

Bluche, T., Ney, H., Louradour, J., \& Kermorvant, C. (2015). Framewise and CTC training of neural networks for handwriting recognition. In 2015 13th international conference on document analysis and recognition (ICDAR) (pp. 81-85). https://doi.org/10.1109/ ICDAR.2015.7333730

Bourlard, H., \& Wellekens, C. J. (1989). Links between Markov models and multilayer perceptrons. In Advances in neural information processing systems (pp. 502-507).

Bourlard, H. A., \& Morgan, N. (1993). Connectionist speech recognition: A Hybrid approach. Norwell, MA: Kluwer Academic Publishers.

Camgoz, N. C, Hadfield, S., Koller, O., \& Bowden, R. (2017). SubUNets: End-to-end hand shape and continuous sign language recognition. In IEEE international conference on computer vision (pp. 22-27).

Cui, R., Liu, H., \& Zhang, C. (2017). Recurrent convolutional neural networks for continuous sign language recognition by staged optimization. In The IEEE conference on computer vision and pattern recognition $(C V P R)$, Honolulu, HI, USA.
Dreuw, P., Deselaers, T., Rybach, D., Keysers, D., \& Ney, H. (2006). Tracking using dynamic programming for appearance-based sign language recognition. In IEEE international conference automatic face and gesture recognition (pp. 293-298). Southampton: IEEE.

Escalera, S., Baró, X., Gonzalez, J., Bautista, M. A, Madadi, M., Reyes, M., et al.. (2014). Chalearn looking at people challenge 2014: Dataset and results. In Computer vision-ECCV 2014 workshops (pp. 459-473). Springer.

Forster, J., Schmidt, C., Hoyoux, T., Koller, O., Zelle, U., Piater, J., et al. (2012). RWTH-PHOENIX-weather: A large vocabulary sign language recognition and translation corpus. In: International conference on language resources and evaluation, Istanbul, Turkey (pp. 3785-3789).

Forster, J., Koller, O., Oberdörfer, C., Gweth, Y., \& Ney, H. (2013a). Improving continuous sign language recognition: Speech recognition techniques and system design. In Workshop on speech and language processing for assistive technologies, Grenoble, France. Satellite workshop of INTERSPEECH 2013 (pp. 41-46).

Forster, J., Oberdörfer, C., Koller, O., \& Ney, H. (2013b). Modality combination techniques for continuous sign language recognition. In Iberian conference on pattern recognition and image analysis. Lecture Notes in Computer Science 7887 (pp. 89-99). Madeira: Springer.

Forster, J., Schmidt, C., Koller, O., Bellgardt, M., \& Ney, H. (2014). Extensions of the sign language recognition and translation corpus RWTH-PHOENIX-weather. In International conference on language resources and evaluation, Reykjavik, Island (pp. 19111916).

Golik, P., Doetsch, P., \& Ney, H. (2013). Cross-entropy vs. squared error training: A theoretical and experimental comparison. In INTERSPEECH (pp. 1756-1760).

Granger, N., el Yacoubi, M. A (2017). Comparing hybrid NN-HMM and RNN for temporal modeling in gesture recognition. In $\mathrm{Neu}$ ral information processing. Lecture Notes in Computer Science (pp. 147-156). Cham: Springer. https://doi.org/10.1007/978-3319-70096-0_16.

Graves, A., \& Schmidhuber, J. (2005). Framewise phoneme classification with bidirectional LSTM and other neural network architectures. Neural Networks, 18(5), 602-610.

Gweth, Y., Plahl, C., \& Ney, H. (2012). Enhanced continuous sign language recognition using PCA and neural network features. In 
CVPR 2012 workshop on gesture recognition, Providence, Rhode Island, USA (pp. 55-60).

Hermansky, H., Ellis, D.W, \& Sharma, S. (2000). Tandem connectionist feature extraction for conventional HMM systems. In 2000 IEEE international conference on acoustics, speech, and signal processing, 2000 (Vol. 3, pp. 1635-1638). ICASSP'00. Proceedings. IEEE.

Huang, J., Zhou, W., Li, H., \& Li, W. (2015). Sign language recognition using 3D convolutional neural networks. In 2015 IEEE international conference on multimedia and expo (ICME) (pp. 16). https://doi.org/10.1109/ICME.2015.7177428.

Jia, Y., Shelhamer, E., Donahue, J., Karayev, S., Long, J., Girshick, R., et al. (2014). Caffe: Convolutional architecture for fast feature embedding. CoRR arXiv:1408.5093.

Koller, O., Forster, J., \& Ney, H. (2015a). Continuous sign language recognition: Towards large vocabulary statistical recognition systems handling multiple signers. Computer Vision and Image Understanding, 141, 108-125. https://doi.org/10.1016/j. cviu.2015.09.013.

Koller, O., Ney, H., \& Bowden, R. (2013). May the force be with you: force-aligned signwriting for automatic subunit annotation of corpora. In IEEE international conference on automatic face and gesture recognition, Shanghai (pp. 1-6).

Koller, O., Ney, H., \& Bowden, R. (2014). Read my lips: Continuous signer independent weakly supervised viseme recognition. In Proceedings of the 13th European conference on computer vision, Zurich (pp. 281-296).

Koller, O., Ney, H., \& Bowden, R. (2015b). Deep learning of mouth shapes for sign language. In Third workshop on assistive computer vision and robotics. Santiago: ICCV.

Koller, O., Ney, H., \& Bowden, R. (2016a). Deep hand: How to train a CNN on 1 million hand images when your data is continuous and weakly labelled. In IEEE conference on computer vision and pattern recognition, Las Vegas, NV, USA (pp. 3793-3802).

Koller, O., Zargaran, S., Ney, H., \& Bowden, R. (2016b). Deep sign: Hybrid CNN-HMM for continuous sign language recognition. In British machine vision conference, York, UK.

Koller, O., Zargaran, S., \& Ney, H. (2017). Re-sign: Re-aligned endto-end sequence modelling with deep recurrent CNN-HMMs. In IEEE conference on computer vision and pattern recognition, Honolulu, HI, USA.

Krishnan, R., \& Sarkar, S. (2015). Conditional distance based matching for one-shot gesture recognition. Pattern Recognition, 48(4), 1298-1310.

Krizhevsky, A., Sutskever, I., \& Hinton, G. E. (2012). ImageNet classification with deep convolutional neural networks. In Advances in neural information processing systems (pp. 1106-1114).

Le, H. S., Pham, N. Q., \& Nguyen, D. D. (2015). Neural networks with hidden Markov models in skeleton-based gesture recognition. In V. H. Nguyen, A. C. Le, \& V. N. Huynh (Eds.), Knowledge and systems engineering. Advances in Intelligent Systems and Computing (Vol. 326, pp. 299-311). Cham: Springer. https://doi.org/ 10.1007/978-3-319-11680-8_24.

LeCun, Y., Bottou, L., Bengio, Y., \& Haffner, P. (1998). Gradientbased learning applied to document recognition. Proceedings of the IEEE, 86(11), 2278-2324.

Marcel, S., Bernier, O., Viallet, J.E, \& Collobert, D. (2000). Hand gesture recognition using input-output hidden Markov models. In Fourth IEEE international conference on automatic face and gesture recognition, 2000. Proceedings (pp. 456-461). IEEE.

Molchanov, P., Gupta, S., Kim, K., \& Kautz, J. (2015). Hand gesture recognition with 3D convolutional neural networks. In Proceedings of the IEEE conference on computer vision and pattern recognition workshops (pp. 1-7).

Neverova, N., Wolf, C., Taylor, G.W, \& Nebout, F. (2014). Multi-scale deep learning for gesture detection and localization. In L. Agapito,
M. M Bronstein, \& C. Rother (Eds.), Computer vision-ECCV 2014 workshops. Lecture Notes in Computer Science (pp. 474490). Springer. https://doi.org/10.1007/978-3-319-16178-5_33.

Ney, H., \& Ortmanns, S. (2000). Progress in dynamic programming search for LVCSR. Proceedings of the IEEE, 88(8), 1224-1240.

Ong, E. J., Koller, O., Pugeault, N., \& Bowden, R. (2014). Sign spotting using hierarchical sequential patterns with temporal intervals. IEEE Conference on Computer Vision and Pattern Recognition (pp. 1931-1938). OH, USA: Columbus.

Pigou, L., van den Oord, A., Dieleman, S., Van Herreweghe, M., \& Dambre, J. (2018). Beyond temporal pooling: Recurrence and temporal convolutions for gesture recognition in video. International Journal of Computer Vision, 126(2-4), 430-439. arXiv:1506.01911.

Rao, Y., Lu, J., \& Zhou, J. (2017). Attention-aware deep reinforcement learning for video face recognition. In Proceedings of the IEEE conference on computer vision and pattern recognition (pp. 39313940).

Richard, A., Kuehne, H., \& Gall, J. (2017). Weakly supervised action learning with RNN based fine-to-coarse modeling. In IEEE conference on computer vision and pattern recognition, Hawaii, USA. arXiv: 1703.08132.

Richard, M. D., \& Lippmann, R. P. (1991). Neural network classifiers estimate Bayesian a posteriori probabilities. Neural Computation, 3(4), 461-483.

Rybach, D., Hahn, S., Lehnen, P., Nolden, D., Sundermeyer, M., Tüske, Z., et al. (2011). RASR-The RWTH Aachen University open source speech recognition toolkit. In IEEE automatic speech recognition and understanding workshop, Waikoloa, HI, USA.

Schlüter, R., Nussbaum-Thom, M., \& Ney, H. (2012). Does the cost function matter in Bayes decision rule? IEEE Transactions on Pattern Analysis and Machine Intelligence, 34(2), 292-301. https:// doi.org/10.1109/TPAMI.2011.163.

Schober, M., Adam, A., Yair, O., Mazor, S., \& Nowozin, S. (2017). Dynamic time-of-flight. In The IEEE conference on computer vision and pattern recognition (CVPR).

Stolcke, A. (2002). SRILM-An extensible language modeling toolkit. In Proceedings on international conference on spoken language processing (ICSLP), Denver, Colorado.

Szegedy, C., Liu, W., Jia, Y., Sermanet, P., Reed, S., Anguelov, D., et al. (2015). Going deeper with convolutions. In The IEEE conference on computer vision and pattern recognition (CVPR), Boston, MA (pp. 1-9).

von Agris, U., Knorr, M., \& Kraiss, K.F (2008a). The significance of facial features for automatic sign language recognition. In 8 th IEEE international conference on automatic face \& gesture recognition, 2008. FG'08 (pp. 1-6). IEEE.

von Agris, U., Zieren, J., Canzler, U., Bauer, B., \& Kraiss, K. F. (2008b). Recent developments in visual sign language recognition. Universal Access in the Information Society, 6(4), 323-362.

Wu, D., Pigou, L., Kindermans, P. J., Le, N., Shao, L., \& Dambre, J. (2016). Deep dynamic neural networks for multimodal gesture segmentation and recognition. IEEE Transactions on Pattern Analysis and Machine Intelligence, 38(8), 1583-1597.

Wu, D., \& Shao, L. (2014). Deep dynamic neural networks for gesture segmentation and recognition. In Computer vision-ECCV 2014 workshops (pp. 552-571). Springer.

Publisher's Note Springer Nature remains neutral with regard to jurisdictional claims in published maps and institutional affiliations. 